\title{
Tuning Magnetic States of Planar Graphene/h-BN Monolayer Heterostructures via Interface Transition Metal-Vacancy Complexes
}

\author{
Bin Ouyang ${ }^{1}$, Jun Song, ${ }^{1, *}$ \\ 1. Department of Mining and Materials Engineering, McGill University, Montreal, QC, Canada
}

\section{SUPPORTING INFORMATION}

\section{S1. Benchmark tests of k-grid, supercell size and exchange-correlation functional.}

Additional calculations using different k-meshes have been performed. Table S1 below lists the calculated energetics and magnetic moment data of the representative Mn- $\mathrm{B}_{\mathrm{SV}}$ system, showing the $\mathrm{k}$-grid of $1 \times 3 \times 1$ is sufficient.

Table S1: Calculated energetics and magnetic moment data using different k-grids for the representative $\mathrm{Mn}-\mathrm{B}_{\mathrm{SV}}$ system.

\begin{tabular}{ccccc}
\hline K-Grid & $1 \times 3 \times 1$ & $3 \times 5 \times 1$ & $5 \times 7 \times 1$ & $7 \times 9 \times 1$ \\
\hline Magnetic moment $\left(\mu_{B}\right)$ & 3.9993 & 3.9995 & 3.9995 & 3.9996 \\
$E_{a}\left(\mathrm{Mn}-\mathrm{B}_{\mathrm{SV}}\right)$ & -6.6415 & -6.6524 & -6.6530 & -6.6531 \\
\hline
\end{tabular}

Meanwhile calculations with different supercell sizes, i.e., $12 \times 6,14 \times 6,12 \times 8$, have been performed. Table S2 below the corresponding energetics and magnetic moment data obtained, again taking $\mathrm{Mn}-\mathrm{B}_{\mathrm{SV}}$ as the representative. From Table S2, it can be concluded that a supercell size of $12 \times 6$ is sufficient to eliminate the size dependence.

Table S2: Calculated energetics and magnetic moment data using different supercell sizes for the representative Mn-B $\mathrm{B}_{\mathrm{SV}}$ system.

$\begin{array}{llll}\text { Supercell size } & 12 \times 6 & 14 \times 6 & 12 \times 8\end{array}$

\footnotetext{
${ }^{*}$ Author to whom correspondence should be addressed. E-Mail: jun.song2@mcgill.ca
} 


\begin{tabular}{cccc}
\hline Magnetic moment $\left(\mu_{B}\right)$ & 3.9993 & 3.9997 & 3.9998 \\
$E_{a}\left(\mathrm{Mn}-\mathrm{B}_{\mathrm{SV}}\right)$ & -6.6415 & -6.6517 & -6.6518 \\
\hline \hline
\end{tabular}

In our DFT calculations, we employed the PBE functional. Test case calculations using other functionals, i.e., DFT $+\mathrm{U}$ and B3LYP, have also been carried out to examine if the results depend on the choice of the exchange correlation functional, with the results shown Table S3. We can see that PBE provides good accuracy in describing the magnetic state and charge distribution. For DFT $+\mathrm{U}$, the Hubbard U-J adapting Dudarev's approach is set as $6.0 \mathrm{eV}$, as reported by previous studies ${ }^{1}$.

Tab. S3: Calculated magnetism of system and calculated charge of atoms $Q_{X}\left(X=N_{1}, N_{2}\right.$, $\mathrm{C}, \mathrm{Mn}$ ) around defect GPBN (atom location indicated in Fig. S1) for different K-grid and supercell, $\mathrm{Mn}-\mathrm{B}_{\mathrm{SV}}$ is chosen as an example.

\begin{tabular}{cccc}
\hline Functional & $\mathrm{PBE}$ & $\mathrm{PBE}+\mathrm{U}$ & $\mathrm{B} 3 \mathrm{LYP}$ \\
\hline Magnetic moment $\left(\mu_{\mathrm{B}}\right)$ & 3.9993 & 4.0000 & 3.9997 \\
$\mathrm{Q}_{\mathrm{N} 1}$ & 3.960 & 3.930 & 3.950 \\
$\mathrm{Q}_{\mathrm{N} 2}$ & 3.941 & 3.915 & 3.947 \\
$\mathrm{Q}_{\mathrm{Mn}}$ & 2.587 & 2.599 & 2.591 \\
\hline
\end{tabular}

Fig. S1 (Color online): Definition of atom locations around Mn- $\mathrm{B}_{\mathrm{SV}}$. 


\section{S2. Adsorption energy definition and van der Waals interactions}
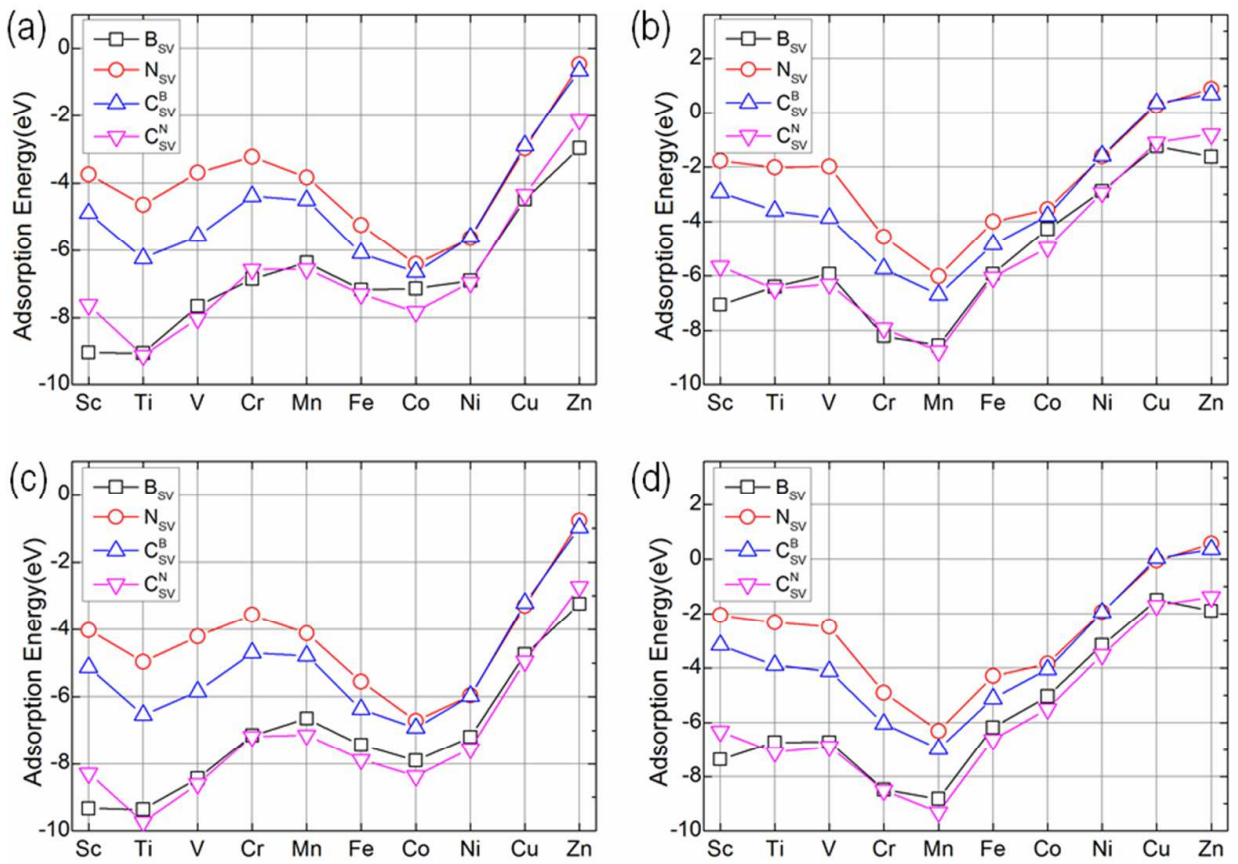

Fig. S2 (Color online): Calculated adsorption energies considering (a) isolated gas metal energy and no vdW correction, (b) metal cohesive energy and no vdW correction, (c) isolated gas metal energy and vdW correction, (d) metal cohesive energy and vdW correction.

The definition of adsorption energy in Eq. 1 takes an isolated transition metal as the reference state. One may also use a stable TM crystal as the reference state in the definition. The resultant adsorption energies from both definitions are presented in Fig. S2, with or without accounting for the van der Waals (vdW) interaction. It is clear from Fig. S2 that the vdW interaction has ignorable effect on the adsorption energetics.

\section{S3. Illustration of out-of-plane displacement due to TM atom adsorption}


(a)

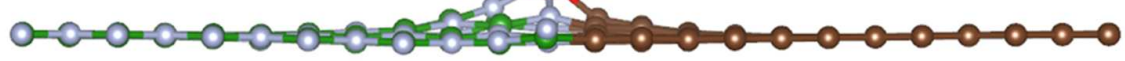

(b)
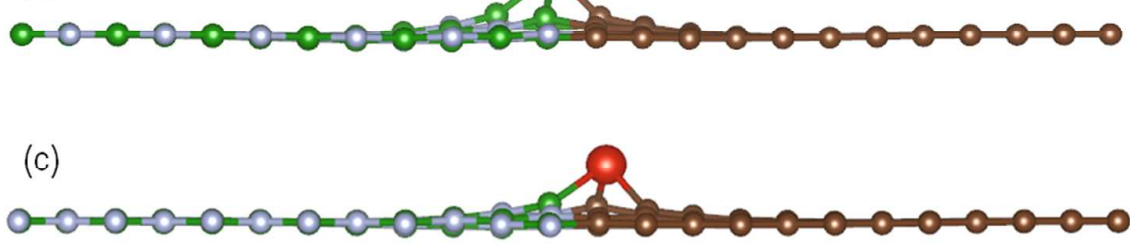

(d)

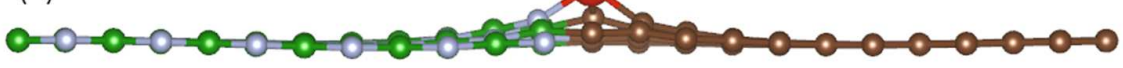

Fig. S3 (Color online): Side view of ground states of (a) $\mathbf{M n}-\boldsymbol{B}_{\boldsymbol{S}}$, (b) $\mathbf{M n}-\boldsymbol{N}_{\boldsymbol{S V}}$, (c) $\operatorname{Mn}-C_{S V}^{B}$, (d) $\mathrm{Mn}-C_{S V}^{N}$.

The TM adsorption leads to appreciable local distortion, and generally certain noticable out-of-plane displacement as shown in Fig. S3 above. 


\section{S4. Side view of buckling strain under compression for $\mathrm{Cr}-\mathrm{N}_{\mathrm{SV}}$}
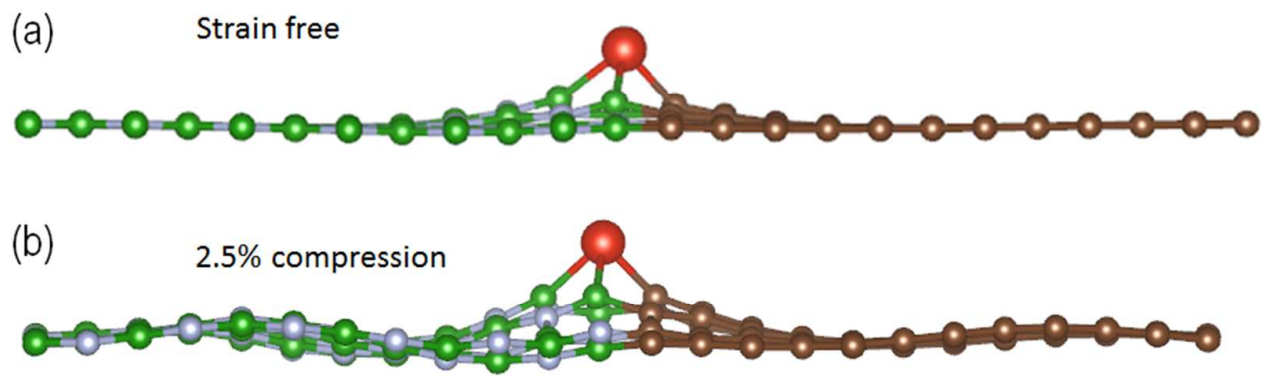

(c)

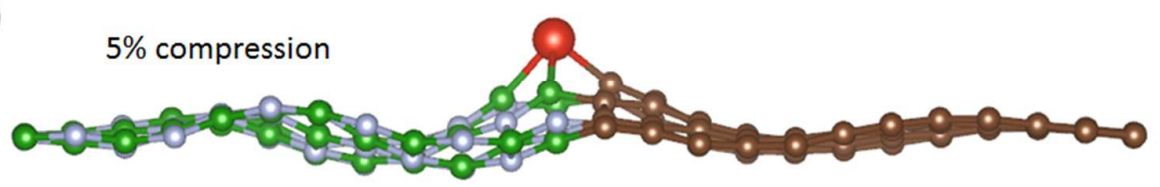

Fig. S 4 Side view of the buckling strain for Cr-NSV under (a) no strain; (b) 2.5\% compression; (c) $5 \%$ compression.

By comparing Cr- $\mathrm{N}_{\mathrm{Sv}}$ system under different compression, it can be inferred that with the increase of compressive strain, the monolayer will gradually buckle. This on one hand release the strain energy of the overall system, on the other hand, the potential bond reconstruction will be enhanced as a result of shrinkage in planar distance. 


\section{Supporting Reference:}

1. C. Franchini, R. Podloucky, J. Paier, M. Marsman and G. Kresse, Ground-State Properties of Multivalent Manganese Oxides: Density Functional and Hybrid Density Functional Calculations. Phys. Rev. B, 2007, $75,195128$. 